\title{
Infecção Hospitalar: Comparação entre Dois Métodos de Vigilância Epidemiológica
}

\author{
Dóra Chor * * Pesquisador do Departamento \\ Carlos Henrique Klein * de Epidemiologia e Metodos \\ Keyla Belizia Feldman Marzochi ** Quantitativos, Ensp/Fiocruz. \\ ** Pesquisadora do Serviço de \\ Epidemiologia, Hospital Evan- \\ dro Chagas/Fiocruz.
}

Uma pequena parcela das infecções hospitalares, cerca de $30 \%$, já é passível de prevençāo através de tecnologia simples e de baixo custo. A vigilância epidemiológica deve servir, portanto, para sugerir $e$ avaliar medidas de controle, além de descrever a freqüência e distribuiçāo das infecçōes. Tendo em vista estes objetivos, questiona-se o método preconizado pelo Ministério da Saúde nos últimos anos e a notificação de infecção preenchida pelos médicos responsáveis pelo atendimento.

$O$ estudo foi realizado no Hospital de Clínicas da Universidade Federal do Paraná, entre março e junho de 1987, onde a notificação foi comparada à busca ativa de casos, utilizada em outros países há 20 anos, e considerada a estratégia de eleiçâo para a vigilância epidemiológica das infeç̧ōes hospitalares. Eficácia e eficiência foram avaliadas em termos do número de casos identificados e do custo de cada um dos métodos. Os resultados foram favoráveis à busca ativa de casos, que pode ser adaptada aos diferentes tipos de hospitais nacionais.

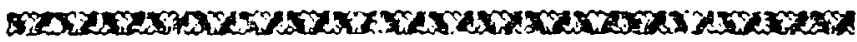

\section{INTRODUÇĀO}

O estudo das infecções hospitalares é um tema novo nas ciências médicas. As infecçōes hospitalares restringem-se àquelas que aparecem durante a internação de pacientes, não estando presentes ou em incubação no momerto da admissão. Aquela denominação não indica, portanto, agente infeccioso específico, nem localizaçāo particular da infecção, mas apenas define o espaço físico onde se inicia.

Cerca de $5 \%$ dos pacientes admitidos em hospitais gerais contraem infecção durante a internação, nos países desenvolvidos (Haley, 1985). No Brasil, apesar de nảo existirem estatísticas nacionais que revelem a magnitude real do problema, estima-se que entre $6,5 \%$ e $15 \%$ dos pacientes internados contraem um 
ou mais episódios de infecção (Ministério da Saúde, 1985), e que entre 50.000 e 100.000 óbitos anuais estejam associados a sua ocorrência (Guimarães, 1985).

As caracteristicas principais destas infecçōes vêm apresentando mudanças de acordo com o tipo de assistência médica prestada em cada período histórico. Assim, a infecção adquirida no meio externo (exógena), mais freqüente nas décadas de 50 e 60 , foi suplantada pela importância de germens oportunistas da própria flora do paciente, que se tomam patogênicos na presença de depressão de seus mecanismos de defesa (Eickhoff, 1969). Esta é determinada pela doença básica ou por agressão diagnóstica e terapêutica relacionada a ampla utilização de procedimentos diagnósticos invasivos e terapêuticas imunodepressoras, que passaram a fazer parte da assistência hospitalar a partir dos anos 70 .

Portanto, nenhum hospital está livre das infecçōes adquiridas durante a internação, já que nenhuma intervenção médica disponivel, no momento, é capaz de erradicá-las. Isto porque, além das condutas invasivas utilizadas adequada ou inadequadamente, existem fatores de risco considerados "inalteráveis" como o envelhecimento da população de internados e, por conseqüência, maior prevalência de doenças imunodepressoras, diagnósticos secundários, necessidade de cirurgias extensas e outros (Freeman, 1981). No entanto, a diminuição do número de casos, ou seja, o controle do agravo é possível e constitui o objetivo dos vários programas de controle existentes em diversos países.

No sentido de identificar as estratégias de controle comprovadamente eficazes, é importante diferenciar entre a parcela de infecção hospitalar passível de prevenção, cerca de $30 \%$ do total (Haley, 1985), das infecçöes não-preveníveis, que ainda constituem a maior parte das ocorrências.

As infecções não-preveníveis são aquelas que ocorrem apesar das condutas e procedimentos serem adequados (Brachman, 1986). Acometem, em geral, pacientes com mecanismo de defesa alterado; são principalmente causadas pela flora endógena, e o modo de transmissão não é estabelecido facilmente (Stamm, 1978). Por outro lado, as infecções preveníveis estão, em geral, relacionadas ao uso de equipamentos ou procedimentos específicos e apresentam em sua origem algum evento que poderia ter sido diferente ou alterado, evitando, assim, seu aparecimento (Wenzel, 1981). Além disso, costumam ocorrer em pacientes com melhores condiçōes de saúde; apresentam modo de transmissão bem definido; são freqüentemente causadas por microrganismos adquiridos no hospital e os métodos de prevenção são conhecidos. Haley e Garner (1986) 
abordaram a questão da seguinte maneira: "as infecções hospitalares passiveis de prevenção são primariamente causadas por problemas no cuidado ao paciente, como o uso e manutenção de sondas urinárias, catéteres venosos e terapia respiratória, bem como na ausência do hábito de lavar as mãos e a má técnica cirúrgica".

A literatura já demonstrou a ineficácia e até mesmo os efeitos negativos de algumas estratégias de controle, utilizadas até a década de 70 , como a contagem de bactérias do ar, o uso irracional da antibioticoprofilaxia e a pesquisa de portadores de microrganismos da flora nomal (Zanon, 1987). Por outro lado, a lavagem ou desinfecção das mãos dos profissionais é tida como a medida mais eficaz na prevenção da infecçāo exógena e de surtos (Reybrouk, 1986). A diminuição do risco de infecção já foi também comprovada através do uso adequado de dois equipamentos: cateter urinário e cateter venoso (Eickhoff, 1975; Goldmann, 1986). Além disso, a eficácia de programas de controle de infecção hospitalar bem estruturados foi comprovada nos Estados Unidos, onde 33\% das infecçōes hospitalares foram prevenidas entre 1970 e 1976 nos hospitais que dispunham de tais programas (Haley, 1985). Assim, a experiência de alguns hospitais nacionais e estrangeiros (Ferraz, 1987; Sharbaugh, 1981) nos autorizam a afirmar que a implantaçăo de medidas de controle é capaz de evitar riscos de doença e óbito, já hoje desnecessários.

$\mathrm{O}$ conhecimento profundo dos mecanismos de defesa do paciente e da epidemiologia das infecçōes em imunossuprimidos, constituem o grande desafio a novas estratégias que possibilitem diminuir ainda mais o número de casos de infecção hospitalar, naquelas instituições que já implantaram todas as normas científicas disponíveis.

$\mathrm{O}$ acompanhamento da freqüência e distribuição das infecções hospitalares, realizado no sentido de permitir a implantação de estratégias de controle adequadas, é executado através da vigilância epidemiológica. A primeira etapa da vigilância é a coleta de informações a respeito da ocorrência das infecções. Esta atividade pode ser desenvolvida de maneiras distintas, sendo a notificação controlada e o método preconizado pelo Ministério da Saúde. Esta vem sendo utilizada no país pela maioria dos hospitais que implantaram programas de vigilância e consiste no preenchimento de fichas especiais pelos médicos responsáveis pelo atendimento, após alta ou óbito do paciente. Vários autores estrangeiros (Eickhoff, 1967; Haley, 1986) e, mais recentemente, alguns nacionais (Évora, 1983; Cardo, 1987) criticam este método, relatando, entre outras deficiências, sua baixa sensibilidade e incapaci- 
dade de detectar situaçōes anormais, já que coleta e analisa dados retrospectivos (após alta ou óbito do paciente). Por outro lado, a metodologia baseada na busca ativa de casos, realizada pelas Comissões de Controle de Infecção Hospitalar (CCIH), grupo especialmente treinado em cada hospital para o controle da infecção, vem sendo apontada, na literatura americana e inglesa, como o método de escolha para a coleta de dados de infecção hospitalar.

Uma vez que grandes esforços vêm sendo empregados no sentido de implantar o controle das infecções hospitalares em todos os hospitais nacionais, desde 1985 , e o único método de coleta de dados indicado pelo Ministério da Saúde vem sendo criticado e foi abandonado por outros países há 20 anos, decidimos realizar uma investigação para comparar a eficácia e a eficiência dos dois métodos, notificação e busca ativa de casos.

\section{METODOLOGIA}

O estudo foi realizado no Hospital de Clínicas da Universidade Federal do Paraná que dispunha de 439 leitos em 1987. Uma vez que a notificação controlada já vinha sendo utilizada desde 1984 , a proposta do estudo foi a implantação da busca ativa de casos em 22 dentre os 35 serviços do hospital (73\% dos leitos), entre março e junho de 1987.

Os dois métodos foram, então, utilizados simultaneamente durante aqueles quatro meses, no sentido de serem comparados o número de pacientes e episódios de infecção hospitalar, o custo total e o custo unitário dos casos diagnosticados por um e por outro método.

O fluxo de dados a partir da notificação foi mantido dentro das normas já estabelecidas, ou seja, as fichas foram preenchidas pelos médicos responsáveis pelo atendimento, após alta ou óbito do paciente, e revisadas pela $\mathrm{CClH}$, através do prontuário.

A busca ativa de casos não foi realizada em todos os pacientes internados, mas apenas em pacientes de alto risco para infecção hospitalar. Estes foram selecionados de acordo com quatro critérios, semelhantes aos utilizados por Wenzel (1976): diagnósticos (leucemia, linfoma, granulocitopenia, diabetes, AIDS, dermatose extensa); resultados de laboratório (hemocultura positiva); procedimentos invasivos (sonda vesical, cateter venoso central, cânula endotraqueal, traqueostomia, cirurgia de grande e médio porte); e tempo de internação (maior ou igual a sete dias). Além dos pacientes portadores de quaisquer destas características, foram também acompanhados todos os internados nas Unidades de Terapia Intensiva e Berçário de Alto Risco. 
Três enfermeiras da Comissāo, assessoradas por um médico, realizaram a busca ativa através de duas visitas semanais, entre $2:$ e $6:$ feira, a cada um dos serviços incluídos no estudo. Nestas unidades, foram registrados em formulário específico os pacientes de al to risco e os casos de infecção hospitalar já identificados. Para classificar os casos como infecção hospitalar, procedeu-se à revisão diária dos resultados de hemoculturas, observação dos pacientes submetidos a procedimentos de risco e revisão de prontuários em busca do registro de resultados de exames laboratoriais, diagnósticos e procedimentos de risco. Os critérios de classificação de infecção hospitalar adotados foram os mesmos utilizados nos casos identificados através da notificação, que são aqueles indicados pelo Ministério da Saúde (1985b).

Foram comparados os egressos portadores de infecção hospitalar de acordo com a notificação com aqueles identificados através da busca ativa, para que a presença ou ausência de infecção, em cada paciente internado no período do estudo, pudesse ser registrada (ver Quadro I).

\section{QUADRO I}

Distribuição dos Egressos de Acordo com a Presença ou Ausểncia de Infecção Hospitalar Segundo Dois Métodos de Coleta de Dados. UC/UFPr. Março a Junho de 1987

\begin{tabular}{|c|c|c|c|c|}
\hline & & \\
\hline & & BUSCA & ATIVA & Total \\
\hline & & + & - & \\
\hline \multirow{2}{*}{ Notificação } & + & 122 & 82 & 204 \\
\hline & - & 190 & 3494 & 3684 \\
\hline \multicolumn{2}{|l|}{ Total } & 313 & 3576 & 3888 \\
\hline
\end{tabular}

A avaliação de eficiência foi realizada através da comparação entre os custos da coleta de dados através de cada um dos métodos, cujos componentes foram os valores da hora-profissional dispendida para sua execução. O tempo necessário para a realização das atividades de notificação e busca ativa foi rigorosamente registrado, possibilitando a comparação do custo total e do custo unitário da identificação de cada caso. 


\section{RESULTADOS}

Considerando-se os quatro meses de estudo, a busca ativa identificou 312 pacientes com infeç̧ão hospitalar, enquanto que, através da notificação, foram diagnosticados 204 pacientes, o que correspondeu a uma diferença de aproximadamente 53\% (Tabela I). Avaliando-se cada serviço isoladamente, este percentual variou amplamente, entre $425 \%$ na Ortopedia e $7,7 \%$ na Neurocirurgia. A busca ativa identificou número muito maior de pacientes do que a notificação em 19 dos 22 serviços estudados, apresentando diferenças importantes em muitas unidades consideradas de alto risco, como Ortopedia, Cirurgia Torácica, Urologia e outros.

É o número de episódios de infecção hospitalar e não o de pacientes infectados que reflete a verdadeira magnitude do problema, já que o mesmo paciente pode apresentar várias infecçôes durante a mesma internação. Em relação aos episódios, (Tabela II), a diferença entre os métodos foi ainda mais importante, já que pôde ser constatado que os médicos, de maneira geral, notificaram apenas um episódio de infecção por paciente. A busca ativa identificou 452 episódos ao longo do estudo, enquanto através da notificação foram registrados apenas 230 , número bastante próximo ao de pacientes com infecção identificados por este método (ver Tabela I). Assim, a busca ativa registrou praticamente o dobro (1,96 vezes) de episódios, variando esta relação entre 6 vezes na Ortopedia e 1,08 vezes no Isolamento Obstétrico.

Realizamos, também, as comparações cruzadas de cada um dos egressos do período, para estabelecer a presença ou ausência de infecção (Quadro I). Foram identificados 122 pacientes com infecção por ambos os métodos; outros 190 pacientes com infeç̧ão foram identificados exclusivamente pela busca ativa, enquanto 82 pacientes foram identificados exclusivamente pela notificação. Os dois métodos não diagnosticaram infecção em 3.494 egressos do período. A diferença entre a proporção de diagnósticos positivos foi estatisticamente significativa $(p<0,001)$, de acordo com 0 teste de McNemar.

No quadro II estão distribuídos os episódios de infecção hospitalar identificados por ambos os métodos. Houve concordância em 154 episódios, 298 episódios foram identificados somente pela busca ativa, enquanto 76 o foram exclusivamente pela notificação. A casela D não foi considerada pẻla imponderabilidade de episódios negativos para ambos os métodos. Esta diferença foi também estatisticamente significativa $(p<0,01)$, pelo teste de McNemar. 


\section{TABELA I}

Pacientes com Infecção Hospitalar de Acordo com

Dois Métodos de Coleta de Dados por Serviço de Registro da In fecção. HC/UFPr.

Março a Junho, 1987

\begin{tabular}{|c|c|c|c|c|c|}
\hline S E R V I ÇO & $\begin{array}{l}\text { Busca } \\
\text { Ativa }\end{array}$ & $\begin{array}{c}\text { Pacientes } \\
\text { (N:) } \\
\text { Notificaçāo }\end{array}$ & $\begin{array}{l}\text { Busca } \\
\text { Ativa }\end{array}$ & $\begin{array}{c}\text { Taxa } \\
(\%) \\
\text { Notificação }\end{array}$ & $\begin{array}{c}\text { Percentual } \\
\text { de Ganho } \\
\text { na Busca } \\
\text { Ativa }\end{array}$ \\
\hline 1. Cirurgia Pediátrica & 5 & 0 & 6,4 & 0 & - \\
\hline 2. Ortopedia & 21 & 4 & 9,7 & 1,9 & $425 \%$ \\
\hline 3. Isolamento & 7 & 2 & 7,6 & 2,2 & $250 \%$ \\
\hline 4. Pediatria & 19 & 8 & 11,2 & 4,7 & $137,5 \%$ \\
\hline 5. 4: Andar Central & 26 & 11 & 8,7 & 3,7 & $136,4 \%$ \\
\hline 6. Cir. Tórax Cardiovascular & 25 & 12 & 17,1 & 8,2 & $108,3 \%$ \\
\hline 7. Cir. Aparelho Digestivo & 33 & 17 & 16,6 & 8,5 & $94,1 \%$ \\
\hline 8. Urologia & 29 & 15 & 24,6 & 12,7 & $93,3 \%$ \\
\hline 9. Clínica Médica Feminina & 19 & 11 & 6,6 & 3,8 & $72,7 \%$ \\
\hline 10. UTI Pediátrica & 5 & 3 & 26,3 & 15,8 & $66,7 \%$ \\
\hline 11. Ginecologia & 10 & 6 & 4,6 & 2,8 & $66,7 \%$ \\
\hline 12. UTI & 11 & 7 & 6,4 & 4,1 & $57,1 \%$ \\
\hline 13. Cirurgia Geral & 27 & 18 & 13,0 & 8,7 & $50,0 \%$ \\
\hline 14. Clínica Médica Masculina & 20 & 16 & 6,7 & 5,3 & $25,0 \%$ \\
\hline 15. Berçário Alto Risco & 23 & 19 & 13,1 & 10,9 & $21,1 \%$ \\
\hline 16. Neurocirurgia & 14 & 13 & 15,1 & 14,0 & $7,7 \%$ \\
\hline 17. Emergência Pediátrica & 6 & 6 & 2,7 & 2,7 & 0 \\
\hline 18. Isolamento Obstétrico & 12 & 12 & 10,1 & 10,1 & 0 \\
\hline 19. Emergência & 0 & 17 & 0 & 2,2 & - $100,0 \%$ \\
\hline Outros Serviços** & ... & 7 & ... & $\cdots$ & ... \\
\hline Total & 312 & 204 & 8,0 & 5,2 & $52,9 \%$ \\
\hline
\end{tabular}

* N: de casos Busca Ativa - N: de casos Notificação

N: de casos Notificaçăo

* Casos notificados em serviços excluídos da busca ativa (ver texto)

- Cálculo impossível

... Dado não coletado 


\section{TABELA $\Pi$}

Episódios de Infecçāo Hospitalar de Acordo com Dois Métodos de Coleta de Dados por Serviço de Registro da Infecção. HC/UFPr.

Março a Junho, 1987

\begin{tabular}{|c|c|c|c|c|c|}
\hline S E R V I Ç O & $\begin{array}{l}\text { Busca } \\
\text { Ativa }\end{array}$ & $\begin{array}{c}\text { Episódios } \\
\text { (N:) } \\
\text { Notificaçāo }\end{array}$ & $\begin{array}{l}\text { Busca } \\
\text { Ativa }\end{array}$ & $\begin{array}{c}\text { Taxa } \\
(\%) \\
\text { Notificação }\end{array}$ & $\begin{array}{c}\text { Percentual } \\
\text { de Ganho } \\
\text { na Busca } \\
\text { Ativa }\end{array}$ \\
\hline 1. Cirurgia Pediátrica & 8 & 0 & 10,3 & 0 & - \\
\hline 2. Ortopedia & 30 & 5 & 13,9 & 2,3 & $500,0 \%$ \\
\hline 3. Isolamento & 10 & 2 & 10,9 & 2,2 & $400,0 \%$ \\
\hline 4. UTI & 30 & 7 & 17,5 & 4,1 & $328,6 \%$ \\
\hline 5. Pediatria & 30 & 8 & 17,8 & 4,7 & $275,0 \%$ \\
\hline 6. Urologia & 55 & 18 & 46,6 & 15,3 & $205,6 \%$ \\
\hline 7. 4: Andar Central & 31 & 11 & 10,4 & 3,7 & $181,8 \%$ \\
\hline 8. Clin. Médica Feminina & 26 & 12 & 9,1 & 4,2 & $116,7 \%$ \\
\hline 9. Cir. Tórax Cardiovascular & 32 & 15 & 21,9 & 10,3 & $113,3 \%$ \\
\hline 10. Cir. Apar. Digestivo & 42 & 20 & 21,1 & 10,1 & $110,0 \%$ \\
\hline 11. Berçário Alto Risco & 32 & 19 & 18,3 & 10,9 & $68,4 \%$ \\
\hline 12. Cirurgia Geral & 38 & 23 & 18,3 & 11,1 & $65,2 \%$ \\
\hline 13. UTI Pediátrica & 8 & 5 & 42,1 & 26,3 & $60,0 \%$ \\
\hline 14. Ginecologia & 11 & 7 & 5,1 & 3,2 & $57,1 \%$ \\
\hline 15. Clínica Méd. Masculina & 27 & 18 & 9,0 & 6,3 & $50,0 \%$ \\
\hline 16. Emergência Pediátrica & 8 & 6 & 3,6 & 2,7 & $33,3 \%$ \\
\hline 17. Neurocirurgia & 21 & 17 & 22,6 & 18,3 & $23,5 \%$ \\
\hline 18. Isolamento Obstétrico & 13 & 12 & 10,9 & 10,1 & $8,3 \%$ \\
\hline 19. Emergência & 0 & 18 & 0 & 2,4 & $-100,0 \%$ \\
\hline Outros Serviços ** & 0 & 7 & ... & $\cdots$ & ... \\
\hline Total & 452 & 230 & 11,6 & 5,9 & $96,5 \%$ \\
\hline
\end{tabular}

* N: de casos Busca Ativa - N: de casos Notificação

$\mathrm{N}$ : de casos Notificação

* * Casos notificados em serviços excluf́dos da busca ativa (ver texto)

- Cálculo impossível

... Dado nảo coletado 


\section{QUADRO II}

Distribuição dos Episódios de Infecção Hospitalar Segundo Dois Métodos de Coleta de Dados. HC/UFPr. Março a Junho, 1987

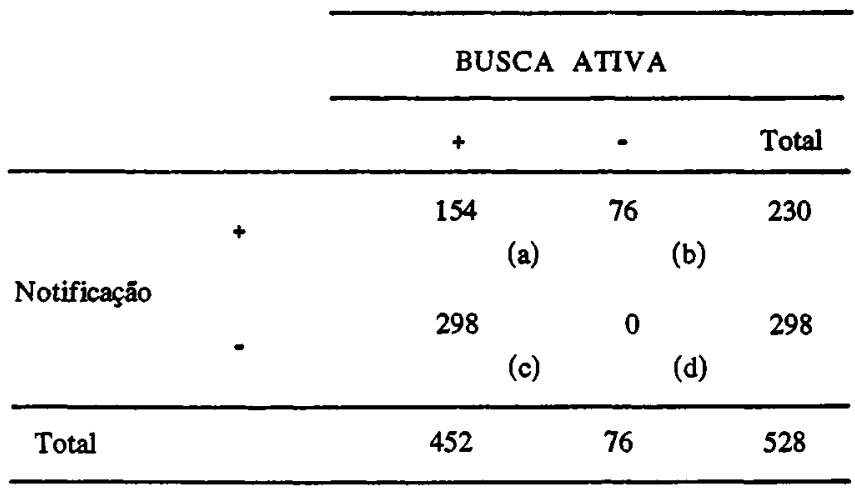

Considerando-se como verdadeiros os diagnósticos positivos segundo ambos os métodos, 528 episódios de infecção hospitalar foram identificados pela busca ativa e/ou pela notificação (ver Quadro II, caselas $a+b+c)$. Destes, a busca ativa identificou 452 , - que representou sensibilidade de $86 \%$. A notificação identificou, por outro lado, 230 episódios do total, com sensibilidade de $44 \%$.

De acordo com o Quadro III, 67 horas mensais foram utilizadas, em média, nas seguintes atividades da notificação: preenchimento, controle e elaboração de relatório. Portanto, excluiu-se, pela dificuldade de medida, o tempo necessário para que o médico estabeleça o diagnóstico de infecção. Dentre aquelas atividades, o controle da ficha, executado por médico da $\mathrm{CCIH}$, consumiu maior parte do tempo, 40 horas (60\%). O custo total da notificação, por mês, foi de 231 dólares, e o custo da identificação de cada episódio de infecção hospitalar foi de 4,1 dólares.

As atividades da busca ativa, ou seja, seleção de pacientes de alto risco, acompanhamento, diagnóstico de infecção hospitalar, revisão dos casos e elaboração de relatório, foram executadas em 135 horas, em média, por mês (Quadro IV). A seleção e o acompanhamento de pacientes nas enfermarias consumiram cerca de $90 \%$ deste total. O custo total da busca ativa foi de 756 dólares, enquanto o custo de cada episódio identificado foi de 6,7 dólares. 


\section{QUADRO III}

\section{Custo Total e Custo Unitário dos Episódios de Infec- ção Hospitalar Identificados pela Notificação Contro- lada $\mathrm{HC} / \mathrm{UFPr}$. Março a Junho, 1987}

- Média mensal de episódios de I. H. detec$\operatorname{tados}(1)$
- Média mensal de horas-médico e valor para preenchimento da notificação

$\mathrm{N}:$ (2) VALOR (3)
- Média mensal de horas-médico e valor para controle da notificação
- Media mensal de horas-médico e valor para relatório

Custo Total $=(3)+(5)+(7)=$ US $\$ 231(8)$

Custo Unitário $=(8) /(1)=$ US $\$ 4,1$

(1) 229 epis6dios $=57$ episódios

$$
4 \text { meses }
$$

(2) Média mensal de 510 notificaçōes positivas e negativas

$10 \%$ em média são positivas $=51$

Média mensal de notificaçōes negativas $=459$

Tempo de preenchimento positiva $=4^{\prime}$

Tempo de preenchimento negativa $=2^{\prime}$

Tempo total de preenchimento* $\left(51 \times 4^{\prime}\right)+\left(459 \times 2^{\prime}\right)=19$ horas

(3) Salário médio de residente no perído: $\mathrm{C} z \$ 13.500,00$

Média mensal de horas trabalhadas: 200 horas

$$
\begin{aligned}
& 1 \text { hora-residente: } \frac{13,500}{200}=C z \$ 67,50 \\
& \text { valor mensal: } 67,50 \times 19=C z \$ 1.282,50 \text { (US\$ 44) }
\end{aligned}
$$

(4) 2 horas $/ \mathrm{dia}=40$ horas $/ \mathrm{mês}$

(5) Salário médio do médico no períado: $\mathrm{Cz} \$ 9.000,00$

Média mensal de horas trabalhadas: 80 horas

1 horamédico: $\underline{9.000}=\mathrm{Cz} \$ 112,50$

$$
80
$$

Valor mensal: $112,50 \times 40=\mathrm{Cz} \$ 4.500,00$ (US\$156)

(6) 8 horas/més

(7) $\mathrm{Cz} \$ 112,50 \times 8=\mathrm{Cz} \$ 900,00$ (US\$ 31) 


\section{QUADRO IV}

\section{Custo Total e Custo Unitário dos Episódios de Infecção Hospitalar Identificados pela Busca Ativa de Casos. HC/UFPr. Março a Junho de 1987}

- Média mensal de episódios de I. H, detectados (1)
- Média mensal de horas-enfermeira e valor para seleçåo e acompanhamento
- Média mensal de horas-médico e valor para revisão
- Média mensal de horas-enfermeira e valor para relatório

N: (2) VALOR (3) N: (4) VALOR (5) N: (6) VALOR (7)

Custo Total $=(3)+(5)+(7)=$ US\$ $756(8)$

Custo Unitário $=(8) /(1)=$ US $\$ 6,7$

(1) 452 episódios = 113 episódios

$$
4 \text { meses }
$$

(2) 2 horas/dia/enfermeira -3 enfermeiras 120 horas/mês

(3) Salário médio da enfermeira no perfodo: $\mathrm{Cz} \$ 20.000,00$

Média mensal de horas trabalhadas: 120 horas

$$
\begin{aligned}
1 \text { hora-enfermeira: } \begin{aligned}
20.000 & =\mathrm{Cz} \$ 167,00 \\
120 &
\end{aligned} \\
\text { valor mensal: } 167,00 \times 12=\mathrm{Cz} \$ 20.000,00 \text { (US } \$ 693 \text { ) }
\end{aligned}
$$

(4) 3 horas/semana - 12 horas/més

(5) Valor da hora-médico: Cz\$ 112,50

Valor mensal: $112,50 \times 12=1.350,00$ (US\$ 46)

(6) 3 horas/més

(7) Valor da hora-enfermeira: $\mathrm{Cz} \$ 167,00$

Valor mensal: $167 \times 3=\mathrm{Cz} \$ 501,00$ (US\$17) 
O custo da identificação de cada episódio de infecção hospitalar através da busca ativa foi de 1,6 vezes maior do que o da notificação que detectou, no entanto, cerca de duas vezes menos episódios do que aquele método (Tabela III).

\section{TABELA III}

Comparação entre a Eficácia e o Custo Unitário da Identificação de Episódios de Infecção Hospitalar Através da Busca Ativa e da Notificação

\begin{tabular}{lcc}
\hline Método & Custo Unit. (US\$) & $\begin{array}{c}\text { Episódios } \\
\text { Identificados }\end{array}$ \\
\hline Notificaçăo & 4,1 & 230 \\
Busca Ativa & 6,7 & 452 \\
\hline Diferença & $63,4 \%$ & $96,5 \%$ \\
\hline
\end{tabular}

\section{DISCUSSÃO E CONCLUSŌES}

A comparação entre os dois modelos de vigilância epidemiológica evidenciou os problemas relacionados à notificação de infecção, que vinha sendo utilizada rotineiramente sem que seus resultados objetivos fossem avaliados. $\mathrm{Na}$ verdade, as atividades envolvidas na vigilância estavam totalmente dissociadas das outras tarefas da $\mathrm{CCIH}$, como implantação de normas de controle e treinamento de pessoal. Assim, seja pela pouca confiança que os próprios membros da Comissão depositavam na notificação, seja pelo atraso das informaçōes coletadas retrospectivamente, as atividades da vigilância não serviam para direcionar as açōes de controle da $\mathrm{CCIH}$, deixando de cumprir seu objetivo principal que é o de fornecer informação para que açōes de controle sejam desencadeadas.

O maior número de pacientes e episódios de infecção hospitalar identificados pela busca ativa, neste estudo, confirma os resultados de outros autores (Haley, 1986; Hofherr, 1979; Wenzel, 1976). As diferenças encontradas entre a busca ativa e a notificação podem ser explicadas pelas características específicas de cada método. A busca ativa apresenta maior padronização de critérios, sendo pacientes e resultados de laboratório acompanhados prospectivamente e os da- 
dos coletados por profissionais especialmente treinados. Por outro lado, as limitações da notificação apontadas por MacPherson (1967) foram também detectadas neste estudo: subnotificação, utilização de critérios classificatórios heterogêneos e relutância em preencher quaisquer formulários por parte da equipe técnica do hospital, especialmente médicos.

A subnotificação de casos ocorreu em vários setores, entre os quais as Unidades de Terapia Intensiva (ver Tabela II), onde se concentram pacientes de alto risco para infecção hospitalar, afetando, assim, profundamente os indicadores epidemiológicos globais do hospital. A subnotificação de setores que concentram pacientes graves foi também observada no Hospital São Paulo (Cardo, 1987), onde "as notificaçōes enviadas não representaram a população de maior risco durante o período de estudo".

Não se dispõe de muitos trabalhos que relatem o valor da sensibilidade dos diferentes tipos de coleta de dados utilizados. Nas experiências referidas por Hofherr (1979) e Thobum (1968), a notificação dos médicos identificou $36 \%$ e $64 \%$ das infecções, respectivamente. Por outro lado, a busca ativa apresentou sensibilidade de $68,5 \%$ (Blake, 1980), 70\% (Bimbaum, 1981) e $80 \%$ (Mulholland, 1975), utilizando diversas fontes de dados. As sensibilidades dos métodos em nosso estudo, em relação à identificaçāo de episódios de infecção hospitalar, apresentaram valores semelhantes aos referidos na literatura (ver Quadro II).

Gostariamos de ressaltar que sensibilidade e especificidade (para esta última, os valores são ainda mais raros na literatura sobre infecção hospitalar) são apenas dois entre vários parâmetros que devem ser avaliados para a escolha do método mais adequado a cada hospital. Assim, por exemplo, foram relatados valores relativamente altos de sensibilidade e especificidade do método de revisäo retrospectiva de prontuários (Wenzel, 1976; Haley, 1980), o que não é suficiente para que este seja considerado o método preferido. Mesmo que valores semelhantes sejam encontrados em hospitais nacionais, o que depende principalmente da qualidade dos prontuários, trata-se de uma técnica passiva, limitada à coleta de dados retrospectivos e que, portanto, não estimula a interferência contínua da $\mathrm{CCLH}$ nos serviços e sua contribuição direta para a prevenção e controle das infecçōes.

$\mathrm{O}$ estudo de custo-efetividade foi realizado para comparação de resultados do mesmo tipo, número de pacientes e episódios de infecção hospitalar, obtidos através dos dois métodos. As outras consequeências prováveis da identificação precoce de um maior número de casos, esperadas somente através da busca ativa,

Cadernos de Saúde Pública, RJ, 6 (2) : 201-217, abr/jun, 1990 
como a redução do consumo de antibióticos, redução das taxas de morbidade e mortalidade por infecção hospitalar e redução do tempo de internaçẫo, não foram objeto de nossa investigação.

A análise do Quadro III revela que 60\% das horas necessárias para as atividades de notificação foram gastas no Serviço de Documentação Médica, para o preenchimento de fichas de notificação que chegaram em branco à $\mathrm{CCIH}$ por não terem sido preenchidas nos respectivos serviços. Por outro lado, $90 \%$ do tempo da busca ativa foram utilizados nas enfermarias (Quadro IV), não sendo possivel separar o número de horas destinadas exclusivamente à coleta de dados, já que outras atividades foram realizadas simultaneamente. Assim, o tempo dispendido na coleta de dados por cada método deve ser avaliado, levando-se em consideração que, enquanto a notificação exigiu o trabalho diário de um médico, altamente especializado, para o preenchimento das fichas com informações retrospectivas, as enfermeiras da busca ativa, além de terem coletado dados prospectivos, realizaram treinamento, responderam dúvidas dos serviços e auxiliaram na implantação de normas de prevenção e controle de infecção. Esta situação foi descrita, também, nos resultados do Projeto SENIC (Study on the Efficacy of Nosocomial Infection Control), nos Estados Unidos, onde foi observado que as enfermeiras que ocupavam mais horas semanais com a coleta de dados eram as que também desenvolviam outras tarefas em maior número: elaboração e implantação de normas, treinamento em serviço, consultorias intemas e investigaçāo de surtos (Emori, 1981).

O tempo dispendido com a busca ativa pode ser, no entanto, bastante reduzido com o aperfeiçoamento da identificação do grupo de alto risco. O critério de inclusão de todos os pacientes hospitalizados por mais de sete dias fez com que muitos pacientes, sem outros riscos, fossem incluídos no estudo. E possível que a probabilidade de infecção seja significativamente maior após período mais longo de internação, como observaram Freeman e McGowan (1981). Estes autores relataram que, no Hospital de Boston, o risco de infecção hospitalar elevou-se de praticamente zero no primeiro dia, a um máximo entre a terceira e a sexta semana de internação.

A diferença entre o custo total dos métodos, 525 dólares, foi irrisória, se verificarmos que representou $61 \%$ do custo médio da intemação de um único paciente no $\mathrm{HC} / \mathrm{UFPr}$, no período correspondente, estimado em 860 dólares. Por outro lado, torna-se vantajosa quando comparada aos resultados quantitativos favoráveis à busca ativa. Considerando-se também os benefícios indiretos, como a capacidade da busca ativa de 
diagnosticar e tratar precocemente a infecção, e seu maior potencial em garantir a execução de medidas de prevenção de novos episódios no mesmo ou em outros pacientes, esta diferença toma-se ainda menor frente aos custos crescentes da hospitalização. Ao contrário, o menor custo da notificação, cujos resultados mostraram-se, mais uma vez, insuficientes no sentido de contribuir para o melhor controle e prevenção da infecção hospitalar, representa um ônus descabido.

A busca ativa de casos já é uma realidade em pelo menos três hospitais nacionais: Hospital de Clínicas da Universidade Federal do Paraná, Hospital de Clínicas da Universidade Federal de Pernambuco e no Hospital São Paulo. É, portanto, um método viável em nosso país, podendo ser adaptado à realidade de instituições de pequeno porte e com características diferentes daquelas apresentadas por hospitais-escola. Em função destas experiências e dos resultados deste estudo, as normas de vigilância do Ministério da Saúde foram revistas, passando a incluir a busca ativa de casos. Assim, muitos hospitais que optaram pelo abandono do programa de controle de infecção em função da ineficácia da notificação podem ter acesso à metodologia de trabalho alternativa.

Não se pode perder a perspectiva de que a finalidade principal da vigilância epidemiológica é a prevenção e o controle das infecções hospitalares, e não a simples elaboração de índices sem finalidade determinada. Acreditamos que as vantagens específicas dos métodos ativos de coleta de dados, que incluem realização de treinamento em serviço, implantação de normas de prevenção, controle e detecção precoce de casos e surtos, entre outras, devem ser consideradas com prioridade na busca do método mais eficaz e eficiente.

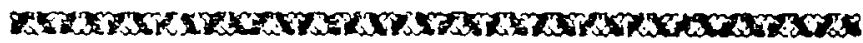

About $30 \%$ of hospital-acquired infections can already be prevented using simple and low cost technology. Therefore, surveillance should be used to suggest and evaluate control measures, as well as to describe infection frequency and distribution. Keeping in mind these objectives, we put into question the method proposed by the Ministry of Health in recent years, infection notification by physicians responsible for care provision.

The study was carried out at the Parana Federal University Hospital, from March to June 1987, where notification was compared to active case-finding, used

Cadernos de Saúde Pública, RJ, 6 (2) : 201-217, abr/jun, 1990 
in other countries for 20 years and considered the optimal strategy for hospital-acquired infection surveillance. Both efficacy and efficiency were evaluated in terms of number of identified cases and method cost. Results were favorable to active case-finding, which can be adjusted to different types of national hospitals.

\section{REFERÊNCIAS BIBLIOGRÁFICAS}

BIRNBAUM, D. e KING, L. A. Disadvantages of Infection Surveillance by Medical Record Chart Review. Am. J. Infect. Cont. 9(1):15-7, 1981 .

BLARE, S. et alii. Surveillance: Retrospective versus Prospective. Am. J. Infect. Cont. 8(3):75-8, 1980.

BRACHMAN, P. S. Epidemiology of Nosocomial Infections. In Bennett, J. V. e Brachman, P. S. Hospital Infections. Boston/ Toronto, Little, Brown and Co. 1986. cap. 1. p. 3-16.

CARDO, D. Comparação entre dois métodos de coleta de dados de infecçẫo hospitalar em Hospital de Ensino. São Paulo, 1987. Tese de Mestrado, Escola Paulista de Medicina.

EICKHOFF, T. C. Hospital Infection Control Begins with Good Surveillance. Hospitals 41:118-20, 1967.

EICKHOFF, T. C. Surveillance of Nosocomial Infections in Community Hospitals I. Surveillance Methods, Effectiveness, and initial results. J. Infecc. Dis. 120(3):305-17, 1969.

EICKHOFF, T. C. Nosocomial Infection. Am. J. Epidemiol. 101(2):93-7, 1975.

EMORI, T. G. et alii. Techniques and Uses of Nosocomial Infection Surveillance in U. S. Hospitals, 1976-1977. Am. J. Med. 70:933-40, 1981.

EVORA, Y. D. M. e ALMEIDA, E. C. S. Comissäo de Controle de Infecçăo Hospitalar de um Hospital-Escola. R. Paul. Hosp. 31(3-4):70-3, 1983.

FERRAZ, E. M. Controle de Infecção Hospitalar: resultados de um estudo prospectivo de dez anos em um hospital universitário. Pernambuco, 1987. Tese para preenchimento do cargo de Professor Titular, Centro de Ciências da Saúde, Universidade Federal de Pernambuco.

FREEMAN, J. e McGOWAN, J. E. Jr. Differential Risks of Nosocomial Infection. Am. J. Med. 70:915-18, 1981.

GOLDMANN, D. A. Nosocomial Infection Control in the United States of America. J. Hosp. Infect. 8:116-28, 1986.

GUIMARÃES, R. X. et alii. Planejamento na Prevenção e Controle da Infecção Hospitalar (1: parte) Laes Haes 6(4):58-61, 1985.

HALEY, R. W. et alii. The Accuracy of Retrospective Chart Review in Measuring Nosocomial Infection Rates. Am. J. Epidemiol. 111(5):516-33, 1980.

HALEY, R. W. et alii. The Efficacy of Infection Surveillance and Control Programs in Preventing Nosocomial Infections in U. S. Hospitals. Am. J. Epidemiol. 121(2):182-205, 1985.

HALEY, R. W. e GARNER, J. S. Infection Surveillance and Control Programs. In Bennett, J. V. e Brachman, P. S. Hospital Infection. Boston/Toronto, Little, Brown and Co, 1986. cap. 3 p. 39-50. 
HALEY, R. W. et alii. Surveillance of Nosocomial Infections. In Bennett, J. V. e Brachman, P. S. Hospital Infection. Boston/ Toronto, Little, Brown and Co, 1986. cap. 3. p. 39-50.

HOFHERR, L. Nosocomial Infection Surveillance Techniques-a review. APIC Joumal 7(3):12-5, 1979.

MACPHERSON, C. R. Practical Problems in the Detection of Hospital-Acquired Infections. Am. J. Clin. Pathol. 50(2):155-59, 1967.

MINISTÉRIO DA SAÚDE. (a) Curso de Introduçāo ao Controle de Infecção Hospitalar. Brasília, 1985.

MINISTÉRIO DA SAÚDE. (b) Manual de Controle de Infecção Hospitalar. Brasflia, 1985.

MULHOLLAND, S, G. et alii. Experience with Detailed Surveillance of Nosocomial Infection. Surg. Gynecol. Obst. 140:941-45, 1975.

REYBROUK, G. Handwashing and Hand Disinfection. J. Hosp. Infect. 8:5-23, 1986.

SHARBAUGH, R. J. An Evaluation of the Efficacy of a Hospital Infection Control Program. Am. J. Infect. Contr. 9(2):35-42, 1981.

STAMM, W. E. Infections Related to Medical Devices. Ann. Intem. Med. 89 (part 2): 764-69, 1978.

THOBURN, R. et alii. Infections Acquired by Hospitalized Patients. Arch. Inter. Med. 121:1-10, 1968.

WENZEL, R. P. et alii. Hospital-Acquired Infections I. Surveillance in a University Hospital. Am. J. Epidemiol. 103(3):251-60, 1976.

WENZEL, R. P. et alii. Identification of Procedure-Related Nosocomial Infections in High-Risk Patients. Rev. Infecc. Dis. 3(4):701-7, 1981.

ZANON, U. et alii. Diretrizes para a Organizaçăo da Comissão de Controle de Infecçỏes Hospitalares. In: Zanon, U. e Neves, J. Infeç̧öes Hospitalares: Prevençäo, Diagnóstico e Tratamento. Rio de Janeiro, Medsi, 1987. cap. 3. p. 47-55. 